J. Clin. Chem. Clin. Biochem.

Vol. 24, 1986, pp. 175-178

(C) 1986 by Walter de Gruyter \& Co.

Berlin - New York

\title{
Circadiane Rhythmik der Konzentrationen von Parathyrin und Calcitonin im Serum
}

\author{
Von M. Radjaipour, E. Kindtner, H. Rösler und M. Eggstein \\ Medizinische Klinik, Abteilung IV (Ärztlicher Direktor: Prof. Dr. M. Eggstein) der Universität Tübingen
}

(Eingegangen am 26. Oktober 1984/2. September 1985)

Zusammenfassung: Die tageszeitliche Abhängigkeit der Konzentrationen von intaktem Parathyrin, von Cterminalen Parathyrin (65 - 84)- und mittregionalen Parathyrin (44-68)-fragmenten, von Calcitonin, Gesamtcalcium, ionisiertem Calcium, Phosphat und Albumin wurde im Serum von fünf Gesunden bestimmt. Intaktes Parathyrin, sein Fragment Parathyrin (44-68) und Calcitonin zeigen einen annähernd synchronen Tagesverlauf mit einem ausgeprägten Peak zwischen $24^{00}$ und $2^{00}$ Uhr morgens. Während Phosphat auch einen deutlichen Tagesrhythmus mit einem Zenit zwischen $1^{00}-8^{00} \mathrm{Uhr}$ zeigt, haben die Gesamtcalcium- und Albuminkonzentrationen im Serum eine abnehmende Tendenz zwischen $20^{00}$ und $6^{00}$ Uhr. Das ionisierte Calcium bleibt im gesamten Tagesverlauf konstant.

\section{Circadian rhythm of parathyrin and calcitonin concentrations in serum}

Summary: The circadian variations of serum intact parathyrin, C-terminal parathyrin (65-84), mid-region parathyrin (44-68) fragments, calcitonin, total calcium, ionized calcium, albumin and phosphate were measured in five healthy subjects. Intact parathyrin, parathyrin $(44-68)$ and calcitonin show a synchronous diurnal fluctuation with a nocturnal increase to a maximum between 24.00 and 2.00 hours. Whereas phosphate has a marked circadian rhythmicity with a zenith between 1.00 and 8.00 hours, total calcium and albumin show a tendency to decrease between 20.00 and 6.00 hours. Ionized calcium concentration remains constant over the whole day.

\section{Einfuihrung}

Der circadiane Rhythmus der Konzentrationen von Parathyrin, Gesamtcalcium (1-4), Albumin und Phosphat $(1-3)$ im Serum von Personen mit normalem Calciumstoffwechsel und Personen mit primärem Hyperparathyreodismus wurde beschrieben.

Jubiz et al. (3) fanden bei Normalpersonen keinen Zusammenhang zwischen dem nächtlichen Abfall des Calciums und der ansteigenden Parathyrinkonzentration im Serum. Ein Calcitonin-Tagesprofil wurde von ihnen nicht bestimmt. Später stellten Hillyard et al. (5) eine tageszeitliche Änderung des Calcitonins im Serum mit einem Maximum nach dem Mittagessen dar. Robinson et al. (6) konnten aber diese Tagesänderung in Seren ihrer fastenden Probanden nicht finden.
Das Ergebnis der ersten Gruppe (5) ist wahrscheinlich, wie Sethi et al. (7) darstellten, ein Stimulationseffekt nach der Nahrungsaufnahme gewesen. Von besonderer Bedeutung ist ein fast paralleler Anstieg der Parathyrin- und Calcitoninkonzentration bei Normalpersonen durch Sekretinstimulation ohne eine Änderung des Calciums im Serum (7).

Vor kurzem haben wir im Tagesprofil eines Kollektivs von 11 Probanden mit normalem Calciumstoffwechsel einen schwachen Anstieg der Konzentration von mittregionalem Parathyrin im Serum gegen Mitternacht beobachtet, während dies beim $\mathrm{C}$-terminalen Parathyrinfragment nicht der Fall war (8). Zur Ergänzung dieser Ergebnisse und in Anbetracht dessen, $\mathrm{da} B$ bezüglich des Calcitonin-Tagesprofils keine ein- 
heitliche bzw. nicht ausreichend zweifelsfreie Darstellung vorliegt, wollten wir folgende Fragen klären:

1. Hat Calcitonin wie Parathyrin einen circadianen Rhythmus?

2. Wo liegt das Maximum der Sekretion?

3. Wie ist der genaue Tagesverlauf von mittregionalem und C-terminalem Parathyrin?

\section{Methodik}

Probanden

Unsere Untersuchungen erfolgten an Personen, die folgende Bedingungen erfüllten:

1. Sie waren klinisch gesund und hatten keine auffäligen Abweichungen der blutchemischen Werte, vor allem der Kenngrößen des Calciumstoffwechsels.

2. Sie durften mindestens 6 Monate lang vor unserer Untersuchung weder an anderen Untersuchungen mit Medikamenteneinnahme teilgenommen, noch regelmäßig Medikamente verabreicht bekommen haben.

3. Sie sollten in dieser Zeit auch regelmäßig nachts geschlafen haben.

4. Sie hatten sich nach einem von uns vorgeschriebenen Speiseplan (ohne Milchprodukte und calciumreiche Getränke) zu ernähren. Die Mahlzeiten erfolgten um $8^{00}, 12^{00}$ und $18^{00}$ Uhr.

5. Sie durften sich während der Untersuchungszeit körperlich nicht anstrengen und sollten sich in der Nacht zur gewohnten Zeit zur Ruhe legen.

Unter diesen Bedingungen konnten wir 5 Personen untersuchen:

a) 3 Personen: 26 - 30 Jahre alt (1 Frau, 2 Männer)

b) 2 Personen: 43 und 47 Jahre alt (1 Frau, 1 Mann)

Die beiden Gruppen wurden bis auf die Blutabnahmezeiten gleich behandelt.

Die Blutentnahme erfolgte bei den 26-30-Jährigen tagsüber bis $24^{00} \mathrm{Uhr}$ alle 4 Stunden, ab $24^{00} \mathrm{Uhr}$ bis $8^{00} \mathrm{Uhr}$ morgens alle 2 Stunden, da in dieser Zeit ein Anstieg von Parathyrin erwartet wurde $(1-4)$. Bei den 43 und 47-Jährigen waren die Blutabnahmezeiten enger, und zwar am Tag alle 2 Stunden und ab $22^{\circ 0}$ Uhr stündlich. Das führte natürlich zu einer erheblichen Störung der Schlafgewohnheiten.

\section{Probenmaterial}

Die Blutentnahme erfolgte aus einer Verweilkanüle. Bei jeder Entnahme wurden $10 \mathrm{ml}$ Blut gewonnen, in Eis gestellt und nach vollständiger Gerinnung 10 Minuten bei $4{ }^{\circ} \mathrm{C}$ zentrifugiert. Das Serum wurde in Portionen zu $1-3 \mathrm{ml}$ aufgeteilt und bei $-20^{\circ} \mathrm{C}$ aufbewahrt.

\section{Bestimmungsmethoden}

Die Bestimmungen der einzelnen Kenngrößen wurden in einer Serie durchgeführt, um die Interassay-Varianz zu umgehen. Die Bestimmung jedes Hormons wurde zweifach durchgeführt.

\section{Intaktes Parathyrin}

Intaktes Parathyrin wurde mit dem $\mathrm{N}^{1}$-tact ${ }^{\mathrm{TM}} \mathrm{PTH}-\mathrm{Kit}$ der Immuno Nuclear Corporation INC (Fa. IBL, D-2000 Hamburg
19) gemessen. Dabei wird intaktes Parathyrin nach einem affinitätschromatographischen Verfahren aus EDTA-Plasma oder Serum extrahiert und radioimmunologisch bestimmt. Nach den Herstellungsangaben und eigenen Versuchen liegt die Wiederfindung von intaktem Parathyrin im Plasma bei $90 \%$, im Serum gut reproduzierbar bei $80 \%$. Wir nahmen diese geringfügig niedrigere Wiederfindung aus Probennahme- und einheitlichen Probenvorbereitungsgründen in Kauf, ińśbesondere, da die Bestimmung der anderen Hormone und blutchemischen KenngröBen durchweg im Serum durchzuführen war. Zur Extraktion des intakten Hormons wurden 2,5 $\mathrm{ml}$ Serum mit 0,5 ml Chicken anti-N-tact-PTH-sepharose-suspension in einer verschließbaren Kunststoffsäule mittels eines Rotors für eine Stunde bei Raumtemperatur gemischt. Danach lief das Eluat aus der Säule und wurde verworfen. Die Säule wurde dreimal mit $1 \mathrm{ml} \mathrm{8,5} \mathrm{g} / 1$ $\mathrm{NaCl}$ gewaschen. Danach wurden das an den Antikörper gebundene intakte Parathyrin und seine $\mathrm{N}$-terminalen Fragmente zweimal mit $0,025 \mathrm{~mol} / 1 \mathrm{HCl}$ eluiert und radioimmunologisch bestimmt. Die Bestimmung von intaktem Parathyrin erfolgte nach einer modifizierten Methode von Lindall et al. (9). Der eingesetzte Antikörper stammte vom Huhn (C-121), Endverdünnung $1: 7500$. Der Tracer bestand aus ${ }^{125}$ I-markiertem Rinder-Parathyrin $(37-84)$. Als Standard diente $\left(\mathrm{Tyr}^{43}\right) \mathrm{Parathyrin}$ (44-68) vom Menschen. Die untere Nachweisgrenze lag bei 3,2 pmol/1 und der Referenzbereich erstreckte sich bis $10 \mathrm{pmol} / 1$. Die VK (Intra- und Interassay) lagen im Konzentrationsbereich von $6,0-7,0 \mathrm{pmol} / \mathrm{l}$ zwischen $6,3 \%$ und $6,7 \%$.

\section{Parathyrinfragmente}

Mittregionales Parathyrin (44-68)-fragment wurde mit dem (44-68) PTH-RIA von Henning, D-1000 Berlin, bestimmt. Der Antikörper vom Schaf (G 2) wurde bei einer Endverdünnung von 1:16000 eingesetzt. Er erfaßte auch das intakte Parathyrin. Als Tracer und Standard wurden synthetisches [ $\left.{ }^{125} \mathrm{I}\right]$ (Tyr ${ }^{43}$ ) Parathyrin (44-68) und Parathyrin (44-68) des Menschen verwendet (10). Der Referenzbereich lag unter $300 \mathrm{ng} / \mathrm{l}$, die Nachweisgrenze bei $80 \mathrm{ng} / \mathrm{l}$. Im Konzentrationsbereich von $80-300 \mathrm{ng} / 1$ fanden wir Variationskoeffizienten von $8 \%$ für Intraassay- und $15 \%$ für Interassaybedingungen. Dabei entsprechen $300 \mathrm{ng} / \mathrm{l}$ Parathyrin $(44-68) 100 \mathrm{pmol} / \mathrm{l}$.

C-terminales Parathyrin (65-84). wurde mit einem modifizierten PTH-Kit der Fa. Mallinckrodt-Diagnostica GmbH ermittelt. Als Antikörper benutzten wir Anti-Rinder-Parathyrin vom Huhn bei einer Endverdünnung von $1: 24000$. Standard und Tracer bestanden aus synthetischem Parathyrin $(65-84)$ und [125I] Parathyrin (65-84) des Menschen. Der Referenzbereich lag unter $650 \mathrm{ng} / \mathrm{l}(300 \mathrm{pmol} / \mathrm{l})$. Die VK lagen in diesem Konzentrationsbereich um 3\% für den Intraassay- und um 7\% für den Interassayansatz. Die Nachweisgrenze lag bei $100 \mathrm{ng} / \mathrm{l}$.

\section{Calcitonin}

Calcitonin wurde mit dem RIA-MAT Calcitonin-Kit (Mallinckrodt) bestimmt. Das Antiserum stammte von Ziegen und wurde mit einem Titer von 1:10000 in diesem Kit eingesetzt. Als Standard diente Calcitonin des Menschen und als Tracer ein synthetisches [ ${ }^{125} \mathrm{I}$ ] Calcitonin. Die Nachweisgrenze lag bei 25 $\mathrm{ng} / \mathrm{l}$. Der Referenzbereich erstreckte sich bis $350 \mathrm{ng} / \mathrm{l}(100$ $\mathrm{pmol} / \mathrm{l})$. In dem Konzentrationsbereich von $25-350 \mathrm{ng} / 1$ lagen die Intra- und Interassay-Variationskoeffizienten um 2,5\% und $10 \%$. Die Radioaktivitätszählung der Ansätze und die Auswertung der Meßergebnisse erfolgten am $\gamma$-Counter (MR 480 der $\mathrm{Fa}$. Kontron Analytik GmbH).

\section{Andere Analyte}

Gesamtcalcium, Albumin und Phosphat wurden am Analysengerät „Monitor Parallel“ (Fa. American-Monitor-Corporation) erstellt (11). Gesamtcalcium, als Cresolphthalein-Komplex bei $575 \mathrm{~nm}$ photometrisch gemessen, besitzt im Referenzbereich $(2,1-2,8 \mathrm{mmol} / 1)$ Variationskoeffizienten in der Serie zwischen $0,9-0,7 \%$. Die Impräzision von Tag'żu Tag war $<1,9 \%$. 
Albumin, nach der Bromcresolgrün-Methode photometrisch bei $600 \mathrm{~nm}$ bestimmt, weist in dem Referenzbereich von $550-739 \mu \mathrm{mol} / 1$ eine analytische Impräzision in der Serie um $2,2 \%$ und von Tag zu Tag $<3,9 \%$ auf.

Phosphat wurde als Phosphomolybdat-Komplex bci $650 \mathrm{~nm}$ ermittelt. In dem Normbereich von 0,8-1,6 mmol/l liegt die Impräzision zwischen $0,6-1,0 \%$ in der Serie und $3,0 \%$ von Tag zu Tag.

Ionisiertes Calcium wurde potentiometrisch mit einem Nova8-Gerät bestimmt. Die Impräzision lag bei einer Konzentration von $1,2 \mathrm{mmol} / 1$ um $0,7 \%$ in der Serie.

\section{Ergebnisse}

In den Abbildungen $1 \mathrm{a}-\mathrm{c}$ und $2 \mathrm{a}$ sind die Konzentrationen von intaktem Parathyrin, Calcitonin, mitregionalem Parathyrin(44-68) und C-terminalem Parathyrin(65-84) im Serum in Abhängigkeit zur Tageszeit dargestellt. Intaktes Parathyrin, Calcitonin

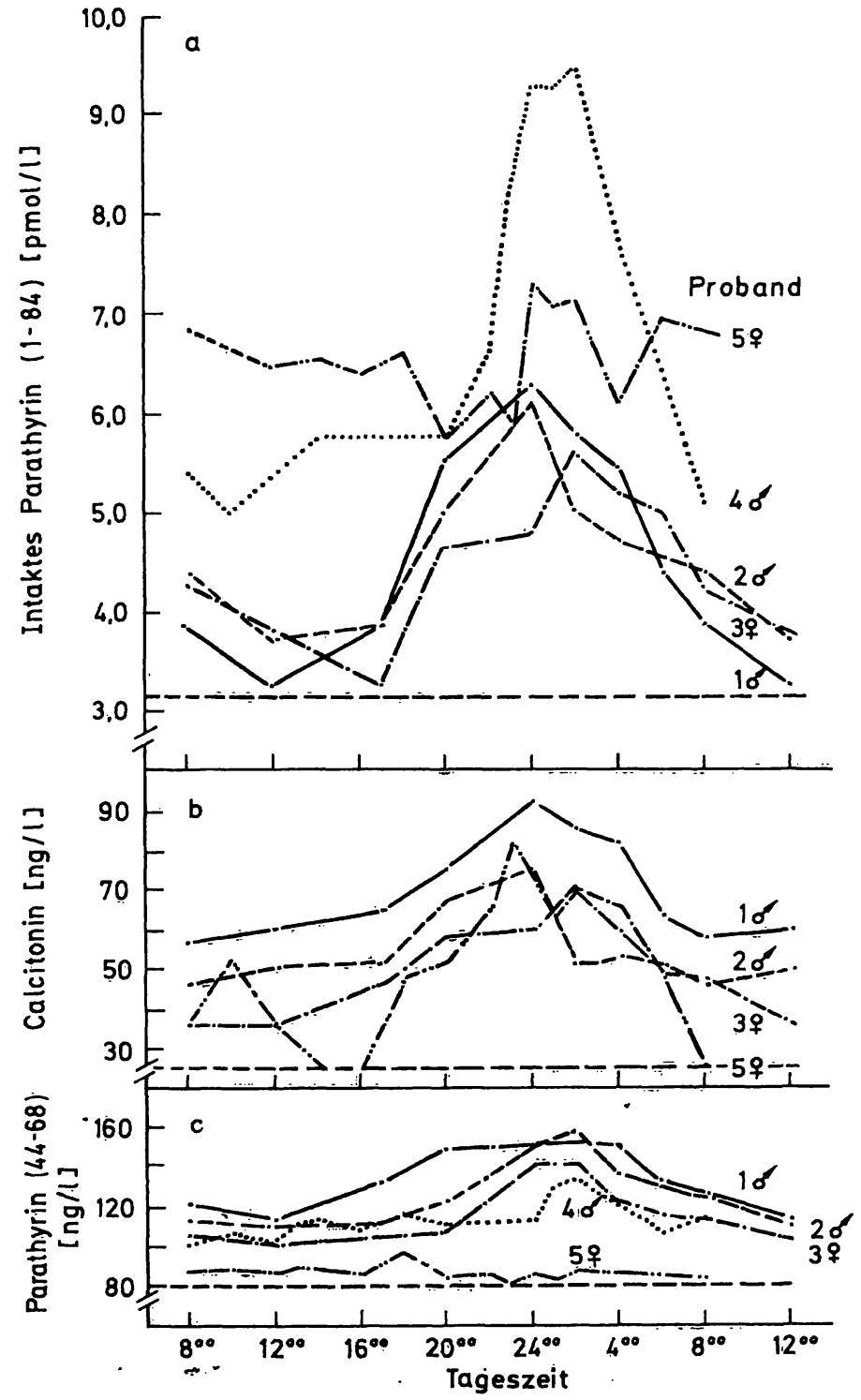

Abb. 1. Tageszeitlicher Verlauf der Konzentrationen von intaktem Parathyrin, Calcitonin und mittregionalem Parathyrin (44-68) im Serum von 5 gesunden Probanden. Die gestrichelten horizontalen Linien zeigen die Nachweisgrenzen.

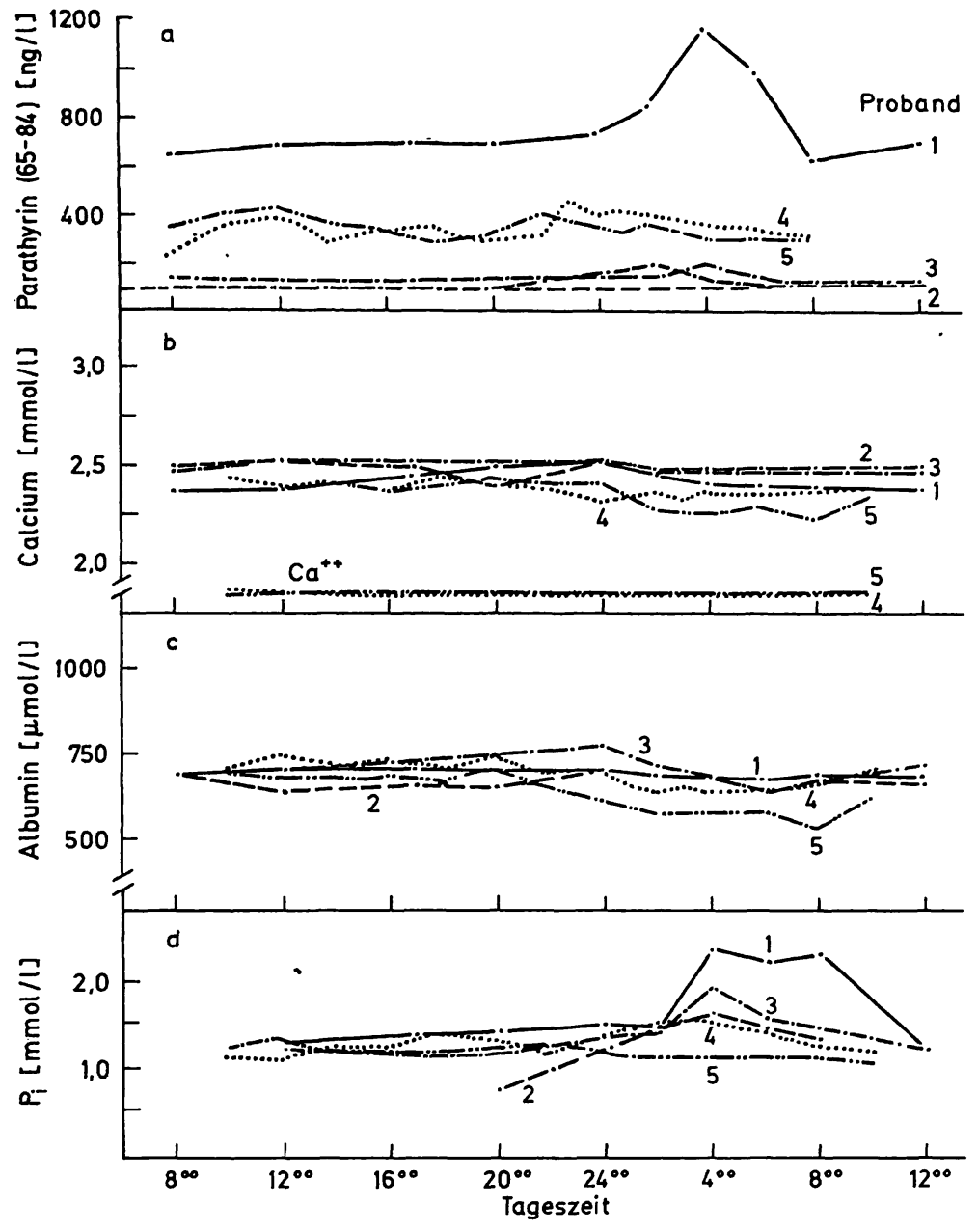

Abb. 2. Tageszeitlicher Verlauf der Konzentrationen von Cterminalem Parathyrin (65-84), Gesamtcalcium, ionisiertem Calcium (s. Text), Albumin und Phosphat im Serum derselben Probanden wie in der Abbildung 1.

und Parathyrin(44-68) zeigen einen annähernd synchronen Tagesverlauf, die Zeit der maximalen Sekretion von intaktem Parathyrin und Calcitonin läßt sich für unsere Probanden zwischen $24^{00}$ und $2^{00}$ Uhr festlegen. Minima und zusätzliche tageszeitliche Änderungen der beiden Hormonkonzentrationen sind individuell unterschiedlich. Vom Minimum ausgehend beträgt der höchste relative Parathyrinanstieg 94\% beim Probanden 1 ( $\delta)$. Bei Probandin 5 (\%) liegt dagegen das Maximum nur um 25\% über dem minimalen Wert. Die Calcitoninkonzentration steigt beim Probanden $1\left(\delta^{\top}\right)$ höchstens um $60 \%$, beim Probanden 5 (ㅇ) dagegen um 228\%, vom Minimum ausgehend, an. Im Serum des Probanden 4 ( $\left.\delta^{3}\right) \mathrm{mit}$ den höchsten Parathyrin-Werten im Tagesverlauf waren die Calcitoninkonzentrationen sehr niedrig und blieben ständig nahe der Nachweisgrenze.

Der Tagesrhythmus von Parathyrin(44-68) (Abb. 1c) zeigt den gleichen Verlauf wie der von intaktem Parathyrin (Abb. 1 a). Die Maximumwerte sind im Vergleich zu den zugehörigen Basiswerten nur zwischen $36 \%$ und $44 \%$ höher. Das Tagesprofil von Parathyrin(65-84) (Abb. 2a) zeigt außer beim Pro- 
banden $1\left(\delta^{*}\right)$, bei dem der Konzentrationsverlauf einen ausgeprägten Peak mit Werten im pathologischen Bereich $(650 \mathrm{ng} / \mathrm{l})$ zwischen $2^{00}-4^{00} \mathrm{Uhr}$ morgens aufweist, keine signifikante Konzentrationsänderung. Auffällig ist, daß dieser Peak sich mit dem zugehörigen Phosphatpeak überlappt (Abb. 2d, Proband 1). Im Gegensatz zu früheren Untersuchungen lassen sich keine signifikanten Maxima im Tagesverlauf der Calciumkonzentration $(1-3,12)$ und Albuminkonzentration $(1-3)$ feststellen (Abb. $2 \mathrm{~b}$ und 2c). Die beobachteten Konzentrationsunterschiede über $24 \mathrm{~h}$ liegen zwischen $4-21 \%$ beim Gesamtcalcium und $2-30 \%$ beim Albumin.

Im Serum von Proband 4 und 5 bestimmten wir zusätzlich noch die Konzentration des ionisierten Calciums. Wie Abbildung $2 \mathrm{~b}$ zeigt, kann die $\mathrm{Ca}^{++}$ Konzentration mit einer Schwankungsbreite von nur 0,07 mmol/1 im Gegensatz zur Gesamtcalciumkonzentration im Tagesverlauf als konstant angesehen werden.

\section{Diskussion}

In Übereinstimmung mit den Ergebnissen anderer Autoren $(1-3)$ fanden wir bei intaktem Parathyrin, das nach affinitätschromatographischer Abtrennung aller nicht $\mathrm{N}$-terminalen Fragmente sehr spezifisch mittels eines mittregionalen Parathyrin-Assays bestimmt wurde, einen Tagesrhythmus mit einer Peakhöhe zwischen $24^{00}$ und $2^{00}$ Uhr. Der geringe zeitliche Unterschied zu der von obengenannten Autoren angegebenen Lage des Maximus $\left(2^{00}-4^{00} \mathrm{Uhr}\right)$ ist wahrscheinlich durch unterschiedliche Lebensgewohnheiten und Schlafrhythmen der Probanden zu erklären. Vorläufige Ergebnisse unserer laufenden Untersuchungen an Schichtarbeitern zeigen diese Verschiebung des Tagesrhythmus. Ähnlich verläuft auch das Calcitonin-Tagesprofil. Sein Peak liegt im Gegensatz

\section{Literatur}

1. Arnaud, C. D., Tsao, H. S. \& Littledike, T. (1971) J. Clin. Invest. 50, $21-34$.

2. Riggs, B. L., Arnaud, C. D., Goldsmith, R. S., Tylor, W. F., McCall, J. T. \& Sessler, A. D. (1971) J. Clin. Endocrinol. Metab. 33, 115-127.

3. Jubiz, W., Canterbury, J. M., Reiss, E. \& Tylor, F. H. (1972) J. Clin. Invest. 51, 2040-2046.

4. Sinha, T. K., Miller, S., Fleming, J., Khairi, R., Edmondsson, J. \& Johnston, C. C., Jr. (1975) J. Clin. Endocrinol. Metab. 41, 1009-1013.

5. Hillyard, C. J., Cooke, T. J. C., Coombes, R. C., Evans, I. M. A. \& Maclntyre, I. (1977) J. Clin. Endocrinol. Metab. 6, 291-298.

6. Robinson, M. F., Body, J. J., Offord, K. P. \& Heath, III. H. (1982) J. Clin. Endocrinol. Metab. 55, 538-544.

7. Sethi, R., Kukrejy, S. C., Bowser, E. N., Hargis, G. K., Henderson, W. J. \& Williams, G. A. (1983) J. Clin. Endocrinol. Metab. 56, 549-552. zu anderen Angaben nicht um die Mittagszeit (5), sondern genau auf dem Peak von intaktem Parathyrin.

Während die Konzentration des Gesamtcalciums im Serum parallel zu der des Albumins in der Zeit von $2^{00}-6^{00} \mathrm{Uhr}$ bei allen Versuchspersonen fallende Tendenz zeigt, bleibt das ionisierte Calcium während $24 \mathrm{~h}$ konstant und damit ohne Tagesrhythmus. Dieses Ergebnis dürfte kaum im Widerspruch zu den Resultaten von Markowitz et al. (13) stehen, da in jener Studie lediglich circadiane Konzentrationsschwankungen des ionisierten Calciums in einer Größenordnung von $0,07 \mathrm{mmol} / 1($ !) bestimmt wurden.

Es ist bekannt, daß die Konzentrationsabnahme des Gesamtcalciums und Albumins auf die Blutdilution in der Nacht zurückzuführen ist (3). Trotzdem bleibt die Konzentration des physiologisch wirksamen ionisierten Calciums konstant. Die Annahme liegt nahe, daß, während das Parathyrin in jedem Augenblick ein Defizit an ionisiertem Calcium auszugleichen versucht, das Calcitonin für die Calciumhomöostase sorgt (14). Befunde an athyreoten Patienten sprechen allerdings dagegen, daß Calcitonin für die Calciumhomöostase wesentlich ist.

Bemerkenswert ist der Tagesrhythmus der Konzentration von mittregionalem Parathyrin (Abb. 1 c). Der Tagesrhythmus des C-terminalen Parathyrinfragments (Abb. 2 a) zeigt nur in einem Fall einen ausgeprägten Peak, der sich nicht mit der tageszeitlichen Schwankung des intakten Parathyrins deckt. Alle Tageswerte dieses Parathyrin-Fragments liegen bei diesem gesunden Probanden an der oberen Grenze des Referenzbereiches (650 $\mathrm{ng} / \mathrm{l}$ ) und steigen bis auf $1200 \mathrm{ng} / \mathrm{l}$ um $4^{00} \mathrm{Uhr}$ morgens an (Abb. 2a).

Für diese und an anderer Stelle beschriebenen (8) Diskrepanzen zwischen den Konzentrationen vom mittregionalen und C-terminalen Parathyrin haben wir derzeit keine befriedigende Erklärung.

8. Radjaipour, M., Rehn, U., Eggstein, M. (1984) Verhandl. der Deutschen Gesellschaft für Innere Medizin 90, $1218-1220$.

9. Lindall, A. W., Elting, J., Ells, J. \& Ross, B. A. (1983) J. Clin. Endocrinol. Metab. 57, 1007-1014.

10. Wood, W. G. (1983) Ärztl. Lab. 29, 311-316.

11. Liebich, H. M., Kuhlmann, E., Radjaipour, M., Maulbetsch, R. \& Eggstein, M. (1985) Labor-Medizin 17-23 und $101-107$.

12. Wisser, H. \& Knoll, E. (1982) Ärztl. Lab. 28, 99-197.

13. Markowitz, M., Rotkin, L. \& Rosen, J. F. (1981) Science $213,672-674$

14. De Luca, H. (1980) Clin. Endocrinol. Metab. 9, 3-25.

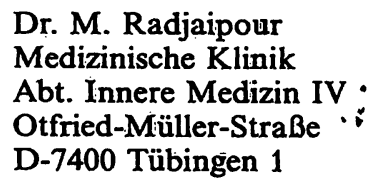

J. Clin. Chem. Clin. Biochem. / Vol. 24, 1986 / No. 3 